Ifeorah et al., Afr., J. Infect. Dis. (2017) 11 (2): 31-38

https://doi.org/10.21010/ajid.v11i2.5

\title{
A COMPARISON OF RAPID DIAGNOSTIC TESTING (BY PLASMODIUM LACTATE DEHYDROGENASE), AND QUANTITATIVE BUFFY COAT TECHNIQUE IN MALARIA DIAGNOSIS IN CHILDREN
}

\author{
Ifeanyi Kanayo Ifeorah ${ }^{1 *}$, Biobele J Brown ${ }^{2}$, Olugbemiro O Sodeinde ${ }^{3}$
} ${ }^{1}$ Nigerian Air Force; Nigerian Air Force Hospital, Bill Clinton Drive Abuja, Nigeria. ${ }^{2}$ University of Ibadan,
University College Hospital Ibadan; ${ }^{3}$ Adjunct Professor,Department of Paediatrics, College of Medicine,
University of Ibadan, University College Hospital, Ibadan.

Corresponding Author Email: fikky17@yahoo.com, biosbrown@yahoo.com, gmgbs@hotmail.com

\begin{abstract}
Background: The World Health Organization (WHO) considers early and rapid diagnosis as one of the strategies to control malaria. This study compared the performance of Quantitative Buffy Coat (QBC) test and the Plasmodium lactate dehydrogenase (pLDH) rapid diagnostic test (RDT) with microscopy as the gold standard.

Materials and Methods: The study involved children ages 0-5 years who presented with a history of fever at the University College Hospital, Ibadan, Nigeria. Blood was collected from each patient and used for RDT, QBC and Giemsastained blood films for malaria parasites (MP). Results of QBC and RDT were compared with microscopy results for the diagnosis of malaria.

Results: A total of 370 cases (194 boys and 176 girls) were studied giving a male: female ratio of 1.1:1. Of the 370 cases tested using Giemsa-stained thick blood films for MP, $78(21 \%)$ were positive. For the QBC test, $78(21 \%)$ of the cases were positive with sensitivity, specificity, positive and negative predictive values of $70.5 \%, 92.1 \%, 70.5 \%$ and $92.1 \%$ respectively. Seventy-six (20\%) of the cases were positive by RDT with sensitivity, specificity, positive and negative predictive values of $84.2 \%, 95.2 \%, 82.1 \%$, and $95.9 \%$ respectively. There was no significant difference in the sensitivity of QBC compared with the RDT.

Conclusion: Both the QBC and the pfLDH (RDT) performed reasonably well in this study Malaria rapid diagnostic tests are recommended in malaria endemic clinical settings to avoid unnecessary antimalarial treatment.
\end{abstract}

Keywords: malaria, quantitative buffy coat, rapid diagnostic testing

List of Abbreviations: AO: Acridine orange, AIDS: Acquired immunodeficiency syndrome, ACT: Artemisinin-based combination therapy, CM:Cerebral malaria, BCP:Benzothiocarboxypurine, DDT:Dichloro-diphenyl-trichloroethane, DNA:DeoxyriboNucleic Acid, ELAM-1: Endothelial leukocyte adhesion molecule, G6PD: Glucose-6-Phosphate Dehydrogenase, HIV: Human immuno deficiency virus, HRP 2: Histidine Rich Protein 2, ICAM -1: Inter cellular adhesion molecule1, ICER: Incremental cost effectiveness ratio, IL-1: Interleukin -1, IFN-g: Interferon-gamma, IgG: Immunoglobulin G, MP: Malaria parasite, NADP: Oxidised Nicotinamide Adenine Dinucleotide Phosphate, NADPH: Reduced Nicotinamide Adenine Dinucleotide Phosphate, PCV: Packed Cell Volume (haematocrit), P. falciparum: Plasmodium falciparum, PLDH: Plasmodium lactate dehydrogenase, PCR: Polymerase Chain Reaction, PPV: Positive predictive value, QBC: Quantitative Buffy Coat examination, TNF: Tumour necrosis factor, NPV: Negative predictive value, RDT: Rapid diagnostic test, SP: Sulphadoxine -Pyrimethamine, SMA: Severe malarial anaemia, UM: Uncomplicated malaria, USA: United States of America, VCAM-1: Vascular cell adhesion molecule, WBC: White Blood Cell, WHO: World Health Organization.

\section{Introduction}

Malaria is a febrile illness caused by protozoa of the genus Plasmodium transmitted by female anopheles mosquitoes which feed on human blood (WHO, 2010). In spite of concerted past efforts at malaria control, it has remained a global health problem and a threat to the life of about $40 \%$ of the world population (WHO, 2010). Today, the goal of 


\section{Ifeorah et al., Afr., J. Infect. Dis. (2017) 11 (2): 31-38 \\ https://doi.org/10.21010/ajid.v11i2.5}

malaria control is to prevent mortality and reduce morbidity and socioeconomic losses through the World Health Organization global Roll Back Malaria (RBM) initiative. The RBM Strategy included early case detection and prompt treatment, integrated vector management and containment of focal epidemics (Breman et al 2004). The World Health Organization (WHO) considers early diagnosis as the first basic element of the strategy to control the disease (WHO, 2009).

Traditionally, laboratory diagnosis had been made using the Giemsa-stained thick blood film microscopy, which continues to be the gold standard test (WHO, 2000). However, this test, in addition to being time-consuming, requires an experienced microscopist, a well-prepared film and a good quality microscope to achieve good sensitivity (Moody, 2002). Other methods have since been developed; these include methods for the detection of Plasmodia within erythrocytes (fluorescent microscopy, Quantitative Buffy Coat [QBC], dark field microscopy, nucleic acid probes and immunofluorescence), methods for the detection of plasmodial antigens in body fluids (radioimmunoassay, direct enzyme immunoassay) and methods for the detection of anti-plasmodial antibodies in serum (indirect immunofluorescence, indirect enzyme immunoassay, Western blotting) (Murray et al., 2008; WHO, 2009).

Giemsa microscopy and rapid diagnostic tests (RDTs) represent the diagnostics most likely to have the largest impact on malaria control today. These methods, each with characteristic strengths and limitations, together have been noted to represent the best hope for accurate diagnosis as a key component of successful malaria control (Nwuba et al., 2001; Wongsrichanalai et al. 2007; Murray et al. 2008; Shillcutt et al. 2008; Lubell et al. 2008; Parija et al., 2009; Uzochukwu et al., 2009; FMOH, 2011). The diagnostic performance of RDTs has been shown to vary with different locations of study to a statistically significant degree (WHO, 2000). This provides the impetus for local comparative studies of RDTs and microscopy. Furthermore, in Nigeria as a whole most studies evaluated the diagnostic performance of the Histidine-Rich Protein 2 (HRP2) based kits but not the Plasmodium Lactate Dehydrogenase (PLDH) based kits (FMOH, 2011). In addition, few studies have also been done in Nigeria on the diagnostic performance of the QBC test, which has also been shown to vary with locations of study and whose potential as a rapid and sensitive method of malaria diagnosis has been documented (Nwuba et al., 2001; Gaye et al 1999; Moody, 2002; Murray et al., 2008; Wongsrichanalai et al., 2007). Few studies have compared the diagnostic performances of these two methods-PLDH and QBC test- of malaria diagnosis (Nwuba et al. 2001; Wongsrichanalai et al., 2007).

This study was an experimental cross sectional design in which the QBC test was compared with a plasmodium lactate dehydrogenase (pLDH) diagnostic test (OPTIMAL) in the diagnosis of malaria, using Giemsa microscopy as gold standard. The endpoints were sensitivity, specificity, positive predictive value (PPV) and negative predictive value (NPV).

\section{Materials and Methods Study design}

This was an experimental cross sectional design in which the performance of QBC test and a pLDH based RDT were compared in diagnosing malaria infection using Giemsa stained microscopy as gold standard. For each diagnostic method (i.e. QBC and pLDH), the outcome measures were the proportion of cases correctly diagnosed as malaria-positive relative to Giemsa-stained MP result and cases correctly diagnosed as malaria-negative relative to the Giemsa-stained result.

\section{Study location}

The study was carried out in the children outpatient clinic and the children's emergency ward of the University College Hospital (UCH), Ibadan, Oyo State, south-western Nigeria between February 1st and May $31^{\text {st }}, 2013$. The UCH is an 850-bed tertiary hospital that serves as a referral centre for the city of Ibadan in which it is located and other parts of Nigeria depending on the diagnosis. The study population was made up of patients $0-5$ years of age with fever at presentation or a history of fever in the three days prior to presentation, as well as other features of both uncomplicated and severe malaria. Using a malaria prevalence rate in febrile under 5 children, of $56.9 \%$ from a previous study, (Ikeh and Teclaire 2008) a minimum of 362 subjects were required.

\section{Blood Collection Procedure}

Each patient had a finger-prick using the ring finger (or big toe in infants) after thorough cleansing with $70 \%$ alcohol. Thick and thin film blood smears were made. Two millilitres of blood was then withdrawn from each patient and put into a Tri-Potassium Ethylene Di-amine Tetra acetic Acid (K $\mathrm{K}_{3}$ EDTA) containing bottle and was used for Packed Cell Volume (PCV), pLDH rapid diagnostic test for malaria and QBC test. 


\section{Ifeorah et al., Afr., J. Infect. Dis. (2017) 11 (2): 31-38 \\ https://doi.org/10.21010/ajid.v11i2.5}

\section{Data Collection Procedure}

All care-givers of the patients were interviewed using a structured pro-forma. History was obtained from the caregivers and children and complete physical examination was carried out on each patient by one of the investigators. All findings from physical examinations and other relevant information including the results of microscopy tests, RDTs, QBC test, PCV and parasite counts, were recorded on the standardized structured pro-forma.

\section{Laboratory Procedures}

The Giemsa stained blood films were examined by two malaria expert microscopists, one of whom has had years of experience working with an internationally-funded malaria research group (in a bid to reduce observer error) in the laboratory, without reference to the results of RDTs and clinical history of patient. Discordant results for blood film microscopy were reviewed by another expert microscopist. Every person positive for malaria by microscopy was treated with an artemisinin-based combination therapy.

OptiMAL RDTs (Diamed AG, Cressier, Switzerland) were transported refrigerated from Lagos to Ibadan and subsequently kept in a refrigerator and brought to room temperature and opened just before use to protect from humidity. The RDTs (OptiMAL-IT PLDH based test kits) were performed and interpreted according to the manufacturer's instructions by one of the researchers who was blinded to each subject's identity and to each sample's thick film result.

For the QBC technique, blood samples in acridine orange coated heparinised tubes were centrifuged at $13000 \mathrm{rpm}$ for 5 minutes and the area just below the buffy coat examined in situ under a fluorescence microscope to detect the parasitized cells. The parasite nuclei fluoresced bright green, and the cytoplasm appeared yellow-orange.

\section{Data Management and Analysis}

Data was entered into a microcomputer and analysed using the SPSS 20.0 for Windows (Inc., Chicago, USA, 2001). Continuous variable estimates were expressed as mean $( \pm S D)$ or median (inter-quartile range) while categorical variables were expressed in proportions and percentages. Categorical variables were compared using the chi-square test. Statistical significance level was set at $\mathrm{p}<0.05$. The Bayesian theory was used to estimate true positives, false positives, true negatives and false negatives and consequently the cases correctly diagnosed. The efficiency of a test corresponds to the percentage of correct test results. Statistical significance of performance differences between tests was evaluated using the McNemar's test.

\section{Results}

\section{General Characteristics}

There were 370 participants in this study, with ages ranging from 3 days to 60 months and a mean ( \pm standard deviation) of $28 \pm 19.6$ months. Participants aged between 0-12 months accounted for $116(31.4 \%)$. Of the 370 cases, $194(52.4 \%)$ were males and $176(47.6 \%)$ were females yielding a male to female ratio of 1.1:1

\section{Malaria diagnostic test results}

Of the 370 cases tested, 78(21.1\%) were positive for malaria parasite by microscopy, $76(20.5 \%)$ were positive on rapid diagnostic test and $78(21.1 \%)$ were positive on the QBC test.

\section{Sensitivity, Specificity, Positive and Negative Predictive Values of RDT and QBC test Comparison of Blood film for MP and Rapid diagnostic test variables}

Table I: Comparison of Rapid diagnostic test results and Blood film for MP

\begin{tabular}{|c|c|c|c|}
\hline & $\begin{array}{l}\text { Blood film } \\
\text { microscopy } \\
+\end{array}$ & - & Total \\
\hline $\begin{array}{l}+ \\
\text { RDT }\end{array}$ & 64 & 14 & 78 \\
\hline- & 12 & 280 & 292 \\
\hline TOTAL & 76 & 294 & 370 \\
\hline
\end{tabular}


Ifeorah et al., Afr., J. Infect. Dis. (2017) 11 (2): 31-38

https://doi.org/10.21010/ajid.v11i2.5

Based on the results in Table I, the sensitivity of RDT in detecting malaria was $84.2 \%$, the specificity was $95.2 \%$, the positive predictive value $82.1 \%$ and the negative predictive value $95.9 \%$. The test efficiency was $93.0 \%$.

\section{Comparison of Blood film for MP and QBC test variables}

Sensitivity, Specificity, Positive and Negative Predictive Values of RDT and QBC test

\section{Comparison of Blood film for MP and Rapid diagnostic test variables}

Table I: Comparison of Rapid diagnostic test results and Blood film for MP

\begin{tabular}{|c|c|c|c|}
\hline & $\begin{array}{l}\text { Blood film } \\
\text { microscopy } \\
+\end{array}$ & - & Total \\
\hline $\begin{array}{l}+ \\
\text { RDT }\end{array}$ & 64 & 14 & 78 \\
\hline- & 12 & 280 & 292 \\
\hline TOTAL & 76 & 294 & 370 \\
\hline
\end{tabular}

Based on the results in Table I, the sensitivity of RDT in detecting malaria was $84.2 \%$, the specificity was $95.2 \%$, the positive predictive value $82.1 \%$ and the negative predictive value $95.9 \%$. The test efficiency was $93.0 \%$.

\section{Comparison of Blood film for MP and QBC test variables}

Table II: Comparison of QBC test variables and Blood film for MP

\begin{tabular}{|c|c|c|c|}
\hline & $\begin{array}{l}\text { Blood film } \\
\text { microscopy } \\
+\end{array}$ & - & TOTAL \\
\hline+ & 55 & 23 & 78 \\
\hline- & 23 & 269 & 292 \\
\hline TOTAL & 78 & 292 & 370 \\
\hline
\end{tabular}

Based on the results in Table II, the sensitivity of QBC in detecting malaria was $70.5 \%$, the specificity was $92.1 \%$, the positive predictive value $70.5 \%$ and the negative predictive value $92.1 \%$. The test efficiency was $87.6 \%$.

Table III: Paired data layout for comparison of sensitivities of RDT and QBC using blood film microscopy as reference QBC

\begin{tabular}{lccc}
\hline RDT & Positive (1) & Negative (0) & Total \\
\hline Positive (1) & 51 & 13 & 64 \\
Negative (0) & 4 & 10 & 14 \\
\hline Total & 55 & 23 & 78 \\
\hline
\end{tabular}

Using the McNemar test $X^{2}=4.76 ; \quad \mathrm{p}=0.049$

Comparison of the sensitivities of RDT and QBC as shown in Table III did not show any statistically significant difference.

Table IV: The paired data layout for comparison of specificities of RDT and QBC

\begin{tabular}{lccc}
\hline \multicolumn{4}{c}{ QBC } \\
\hline RDT & Positive (1) & Negative (0) & Total \\
\hline Positive (1) & 4 & 8 & 12 \\
Negative (0) & 19 & 261 & 280 \\
\hline Total & 23 & 269 & 292 \\
\hline
\end{tabular}

$X^{2}=4.48, \quad \mathrm{p}=0.052$ 


\section{Ifeorah et al., Afr., J. Infect. Dis. (2017) 11 (2): 31-38 \\ https://doi.org/10.21010/ajid.v11i2.5}

Similarly, comparison of the specificities of RDT and QBC did not show any statistically significant difference (Table IV).

\section{Distribution of malaria parasitaemia according to parasite density}

The Parasite densities of the patients in this study ranged from 31 to 273,831 parasites per microliter with a geometric mean parasite density of $63371.9 \pm 65544.4$. Forty-nine $(64.4 \%)$ of the 76 positive slides for malaria parasites had parasite densities $>4,500$. (Table V).

Distribution of malaria parasitaemia according to parasite density

Table V: Sensitivities of RDT and QBC tests at various parasite densities

\begin{tabular}{|c|c|c|c|c|c|}
\hline $\begin{array}{l}\text { Parasites count } \\
\left(\text { parasites } \mu L^{-1}\right)\end{array}$ & $\begin{array}{l}\text { Thick Blood } \\
\text { film (MP) } \\
\text { Positive }\end{array}$ & $\begin{array}{l}\text { RDT } \\
\text { Positive }\end{array}$ & $\begin{array}{l}\text { RDT Sensitivity } \\
(\%)\end{array}$ & $\begin{array}{l}\text { QBC test } \\
\text { Positive }\end{array}$ & $\begin{array}{l}\text { QBC } \\
\text { sensitivity } \\
(\%)\end{array}$ \\
\hline$\leq 500$ & 8 & 1 & 12.5 & 2 & 25.0 \\
\hline $501-1,000$ & 3 & 1 & 33.3 & 1 & 33.3 \\
\hline $1,001-4,500$ & 18 & 13 & 72.2 & 13 & 72.2 \\
\hline$>4,500$ & 49 & 49 & 100.0 & 39 & 79.6 \\
\hline Total & 78 & 64 & 82.1 & 55 & 70.5 \\
\hline
\end{tabular}

For both RDT and QBC tests, there was a tendency for increased sensitivity with increasing parasite densities with $100 \%$ sensitivity for RDT and $79.6 \%$ for QBC in patients with a parasite count of $>4,500$ per microliter (Table V).

\section{Discussion}

Malaria is a parasitic infection of global importance and a major public health problem in Nigeria, accounting for sizeable morbidity, mortality and economic loss (NPC, 2012; Orimadegun et al., 2007). The absolute necessity for rational anti-malarial therapy in the face of rampant drug resistance places importance on the accuracy of malaria diagnosis (WHO, 2009 ; 2010).

The $70.5 \%$ sensitivity of the QBC test obtained from this study was low, in keeping with the findings from Adeoye and Nga (2007) in Nigeria where a sensitivity of $55.9 \%$ was reported and the sensitivity of $78 \%$ reported by Parija et al. (2009) in South India. Findings from the present study were however at variance with findings from a study by Fagbenro-Beyioku et al. (2007) in Lagos, Nigeria in a study involving 270 patients of various ages, where a sensitivity of 92.5\% was reported (Fagbenro-Beyioku et al., 2007). Studies in other parts of the world have also yielded high sensitivities: Anthony et al. (1992) reported 94\% sensitivity for QBC in the eastern Jayawijaya Mountains of Irian Jaya (Indonesian New Guinea), an area of low parasite transmission, and Ponsilapatip et al. (1990) reported 99.5\% sensitivity in Thailand (Ponsilapatip et al., 1990). The latter studies were conducted in areas of low malaria transmissions with the implications that observed cases were likely to be of high malaria densities thus accounting for the high sensitivities as detection of the parasites would be much easier.

Specificity of the QBC test in this test was $92.1 \%$ which was in keeping with the finding of $95.0 \%$ reported by Wang et al. in a study conducted in Junlian and Mingshan counties, Sichuan, China (Wang X et al., 1996).

Sensitivity and specificity, of Optimal RDT test in the present study were 84.2 and 95.2\% respectively. Furthermore, the positive predictive and negative predictive values were 82.1 , and $95.9 \%$ respectively, which are consistent with findings from earlier studies (Agomo et al., 2003; Chayani et al., 2004). Agomo et al. in a study of 240 patients from South West Nigeria, reported sensitivity, specificity, positive and negative predictive values of 63.95, 92.20, 82.1 and $82.1 \%$, respectively for the OptiMAL RDT (Agomo et al., 2003). Chayani et al. (2004) in their study in India, of 232 cases reported that the OPtiMAL test had sensitivity of $88.4 \%$ and $96.8 \%$ for $P$. falciparum and $P$. vivax respectively (Chayani et al., 2004) Higher sensitivities have been however reported from other studies. Gbotoso et al. (2010) reported a sensitivity of $97.2 \%$ for the Optimal RDT, tests performed on whole blood samples while it was $48.3 \%$ for tests performed on the supernatant of spun saliva (Gbotoso et al., 2010). In that study, patients aged 8 months to 13 years were eligible to participate in the saliva study if they had microscopically confirmed pure $P$. falciparum infection $\geq 2,000$ parasites/microlitre of blood, a temperature $\geq 37.5^{\circ} \mathrm{C}$ or recent history of fever, and absence of other concomitant illness. The selection of patients with such parasite densities apparently led to the high sensitivity obtained from the study in contrast with this present study where parasite counts were as low as 50 parasites/ microlitre. Obviously, one of the reasons for the low sensitivity of detection using Optimal-IT dipsticks may be the fact that this RDT was commercially designed to detect high levels of lactate dehydrogenase in whole blood. It could also be due to the presence of gene mutation variants of 


\section{Ifeorah et al., Afr., J. Infect. Dis. (2017) 11 (2): 31-38 \\ https://doi.org/10.21010/ajid.v11i2.5}

the plasmodium species which express little or no pLDH antigen, thus resulting in a negative pLDH assay (Malik et al., 2004). False positive reactions may have occurred as a result of sequestration of parasites in the deep tissues and organs resulting in negative results on blood film microscopy but positive results by the RDT as the enzymes are present in blood (Makler, 1998; Murray, 2008).

Based on findings of this study, there was no statistically significant difference between the sensitivities and specificities of QBC and pLDH RDT in the diagnosis of malaria. This is in keeping with a similar study done in India by Kocharekar et al. where they found that the sensitivity of pLDH based RDT test was similar when compared to QBC (Kocharekar et al., 2014). In spite of the greater cost or more expensive equipment required (Adeoye et al., 2007) our study showed no superiority of QBC over $\mathrm{pLDH}$, the latter is therefore recommended in preference to QBC in a resource constrained setting like ours.

The sensitivity of the pLDH in this study was highest at parasite densities $>4,500$ parasites/microlitre, whereas the lowest sensitivity was documented at the parasite density less than 500 parasites/microlitre in keeping with the fact that the RDT was commercially designed to detect high levels of parasite lactate dehydrogenase in whole blood. Similar findings have been reported by different researchers. Cooke et al. in a study in 1999, observed similar test performances in terms of sensitivity, specificity, positive as well as negative predictive values, but also observed a marked decline in sensitivity of the test at parasitaemia less than 500 parasites/ $\mu 1$ (Cooke et al., 1999). Similarly, the sensitivity of the QBC test, was related to parasite density and highest at parasite densities $>4,500$ parasites/ microlitre but lowest at the parasite densities less than 500/microlitre in keeping with findings by Lowe et al. in Kenya, where QBC had an overall sensitivity of $93.6 \%$ but 41.7\% sensitivity at parasitaemia less than 100 per microliter (Lowe et al., 1996). A study by Adeoye and Nga in Nigeria also showed a sensitivity of $33.3 \%$ at low parasite densities (overall sensitivity was 55.9\%) (Adeoye and Nga, 2007). The occurrence of false negative pLDH and QBC tests at low parasite densities indicates the need for a high index of suspicion in cases with clinical features in keeping with malaria. Patients that test negative to these screening tests should therefore be subjected to microscopy of stained blood films.

The WHO has recommended a minimum standard of $95 \%$ sensitivity at parasite densities of $100 / \mu \mathrm{L}$. However, in the present study, at parasite densities of $<500 / \mu 1$ the sensitivities were 12.5 percent. This may not be in keeping with the WHO standards, but in terms of parasite threshold associated with fever in high transmission settings, which has been noted by some authors (Smith et al., 1994; Mwangi et al., 2005; Mmbando et al., 2009) to be at parasite densities $\geq 4$, 000 parasites/microliter in children aged $>1$ year to 15 years, the sensitivity at parasite densities $>4,500$ parasites/microliter was $100 \%$ for the pLDH RDT whereas it was 79.6\% for the QBC test (Smith et al., 1994; Mwangi et al., 2005; Mmbando et al., 2009). This implies that in a high transmission setting such as in Ibadan, a negative pLDH test and QBC test in that age range would make the diagnosis of malaria unlikely. They are therefore recommended for use in settings that lack microscopists depending on the resources available after weighing the storage requirements (such as refrigeration and the initial start up high costs of the QBC equipment. Furthermore, pLDH is a very good alternative in settings where expert microscopist (microbiologist) is not available especially in endemic area of tropical African region where level of parasitaemia makes it $100 \%$ sensitive and highly specific too.

A cost-effectiveness analysis comparing the two tests would therefore be useful in deciding which test would be suitable in any setting.

\section{Conclusion}

Malaria diagnosis has remained a useful tool in the strategy for the control of malaria. This study has revealed the usefulness of the RDT and the QBC in the diagnosis of childhood malaria in a resource constrained setting. Sensitivities of both are better at higher parasite densities. The presence of false positives at low parasite densities underscores the need to carry out microscopy in test negative cases when malaria is strongly suspected. The lack of significant difference in sensitivity between the RDT and QBC makes the RDT a better choice given its less expensive requirements. The study further underscores the importance of the use of the RDT tests to confirm diagnosis in resource constrained settings where persons are not trained in microscopy.

\section{Ethical consideration}

Ethical approval for the study was obtained from the Joint Ethics Committee of the University of Ibadan and University College Hospital, Ibadan and informed consent obtained from all caregivers. 


\section{Ifeorah et al., Afr., J. Infect. Dis. (2017) 11 (2): 31-38 \\ https://doi.org/10.21010/ajid.v11i2.5}

\section{Acknowledgement}

I wish to acknowledge $\mathrm{Mr}$ Afolabi (Laboratory Scientist) and the members of the malaria proteomics team for their assistance. I also wish to acknowledge Mrs Patience N Ogunjobi, and Mrs Victoria U Nwachukwu, both of the Department of Medical Microbiology and Parasitology of the University College Hospital who performed the QBC microscopy and Miss Tolu Mumini for the data analysis. Special thanks also go to Mrs IM Ifeorah for all the support.

\section{References}

1. Adeoye GO and Nga IC. Comparison of quantitative buffy coat technique (QBC) with Giemsa -stained thick film for diagnosis of malaria. Parasitol Int., 2007; 56(4):308-12.

2. Agomo PU, Asianya VN, Akindele SK, Agomo CO, Akinyele MO. Investigation of the efficacy of two rapid assessment techniques OptiMAL 1 and SD Bioline for the diagnosis of malaria in rural area of Nigeria. Afr J Clin Exp Microbiol., 2003; 4: 6-13.

3. Anthony RL, Bangs MJ, Anthony JM, Purnomo. On-site diagnosis of Plasmodium falciparum, $P$. vivax, and $P$. malariae by using the Quantitative Buffy Coat system. J Parasitol., 1992; 78(6):994-8

4. Breman JG, Alilio MS, Mills A. Conquering the intolerable burden of malaria: what's new, what's needed a summary.Am J Trop Med Hyg., 2004;71(2):1-15.

5. Chayani N, Das B, Sur M, Bajoria S. Comparison of parasite lactate dehydrogenase based immunochromatographic antigen detection assay (OptiMAL) with microscopy for detection of malaria parasites. Indian J Med Microbiol., 2004; 22(2):104-6.

6. Cooke AH, Chiodini PL, Doherty T, Moody AH, Ries J, Pinder M. Comparison of a parasite lactate dehydrogenase-based immunochromatographic antigen detection assay (OptiMAL) with microscopy for the detection of malaria parasites in human blood samples. Am J Trop Med Hyg., 1999; 60(2): 173-176.

7. Fagbenro-Beyioku AF, Ojuromi OT, Orenaike IK. Qualitative comparison of qualitative buffy coat and light microscopy in malaria diagnosis. Trop Doct; 2007; 37(1):60-1

8. Federal Ministry of Health: Nigerian National Guidelines for Diagnosis and Treatment of Malaria; 2011Federal Ministry of Health, p23.

9. Gaye O, Diouf M, Diallo S. A comparison of thick films, QBC malaria, PCR and PATH falciparum malaria test strip in Plasmodium falciparum diagnosis. Parasite, 1999; 6: 273-275.notfoundintext

10. Gbotoso GO, Happi CT, Folarin O, Keyamo O, Sowunmi A, Oduola AM. Rapid detection of lactate dehydrogenase and genotyping of Plasmodium falciparum in saliva of children with acute uncomplicated malaria. Am J Trop Med Hyg; 2010; 83(3):496-501.

11. Ikeh EI and Teclaire NN. Prevalence of malaria parasitaemia and associated factors in febrile under-5 children seen in Primary Health Care Centres in Jos, North Central Nigeria. Niger Postgrad Med J; 2008 ;15(2):65-9

12. Kocharekar MM, Sarkar SS, Dasgupta D. Comparative study of modified quantitative buffy coat and two rapid tests in comparison with peripheral blood smear in malaria diagnosis in Mumbai, India. J Parasitol Res; 2014; 194651

13. Lowe BS, Jeffa NK, New L, Pedersen C, Engbaek K, Marsh K. Acridine orange fluorescence techniques as alternatives to traditional Giemsa staining for the diagnosis of malaria in developing countries. Trans $R$ Soc Trop Med Hyg; 1996; 90(1):34-6

14. Lubell Y, Hopkins H, Whitty CJ, Staedke SG, Mills A. An interactive model for the assessment of the economic costs and benefits of different rapid diagnostic tests for malaria. Malar J; 2008; 7(1): 21.

15. Makler MT. A review of practical techniques for the diagnosis of malaria. Ann Trop Med Parasitol; 1998; 92(4):419-33.

16. Malik S, Khan S, Das A, Samantaray JC. Plasmodium lactate dehydrogenase assay to detect malarial parasites. Natl Med J India; 2004; 17(5):237-9.

17. Mmbando BP, Lusingu JP, Vestergaard S, Lemnge MM, Theander TG, Scheike TH Parasite threshold associated with clinical malaria in areas of different transmission intensities in north eastern Tanzania. BMC Med Res Methodol BMC series; 2009; 9:75DOI: 10.1186/1471-2288-9-75.

18. Moody A. Rapid diagnostic tests for malaria parasites. Clin Microbiol Rev; 2002; 15(1):66-78.

19. Murray CK, Gasser RA Jr, Magill AJ, Miller RS. Update on Rapid Diagnostic Testing for Malaria. Clin Microbiol Rev; 2008; 21(1): 97-110.

20. Mwangi T, Ross A, Snow R, Marsh K: Case definitions of clinical malaria under different transmission conditions in Kilifi District, Kenya. J Inf Dis; 2005; 191 (11): 1932-1939. 10.1086/430006. 
Ifeorah et al., Afr., J. Infect. Dis. (2017) 11 (2): 31-38

https://doi.org/10.21010/ajid.v11i2.5

21. National Population Commission (NPC) [Nigeria], National Malaria Control Programme (NMCP) [Nigeria], ICF International: Nigeria Malaria Indicator Survey 2010. Abuja, Nigeria: NPC, NMCP, and ICF International; 2012

22. Nwuba RI, Anumudu CI, Omosun YO, Sodeinde O, Nwagwu M. Evaluation of a rapid immunochromatographic card test for Plasmodium falciparum in Ibadan, Nigeria. Afr J Med Sci; 2001; 30(1-2):123-4.

23. Orimadegun AE, Fawole O, Okereke JO, Akinbami FO, Sodeinde O. Increasing burden of childhood severe malaria in a Nigerian tertiary hospital: implication for control J Trop Pediatr; 2007; 53(3): 185-189.

24. Parija SC, Dhodapkar R, Elangovan S, Chaya DR. A comparative study of blood smear, QBC and antigen detection for diagnosis of malaria. Indian J. Pathol Microbiol; 2009; 52(2): 200.

25. Ponsilapatip J, Namsiripongpun V, Wilde H, Hanvanich M, Chtivongse S. Detection of Plasmodia in acridine orange stained capillary tubes. Southeast Asian J Trop Med Public Health; 1990; 21(4):534-40.

26. Shillcutt S, Morel C, Goodman C, Coleman P, Bell D, Whitty CJM. Cost-effectiveness of malaria diagnostic methods in sub-Saharan Africa in an era of combination therapy. Bull World Health Organ; 2008; 86(2): 101-110.

27. Smith T, Armstrong Schellenberg J, Hayes R. Attributable fraction estimates and case definitions for malaria in endemic areas. Statistics in Medicine. 1994, 13 (22): 2345-2358. 10.1002/sim.4780132206Chandler C, Drakeley $\mathrm{C}$, Reyburn H, Carneiro I. The effect of altitude on parasite density case definitions for malaria in north-eastern Tanzania. Trop Med Int Health; 2006, 11 (8): 1178-1184. 10.1111/j.1365-3156.2006.01672.x.

28. Uzochukwu BS, Obikeze EN, Onwujekwe OE, Onoka CA, Griffiths UK. Cost-effectiveness analysis of rapid diagnostic test, microscopy and syndromic approach in the diagnosis of malaria in Nigeria: implications for scaling-up deployment of ACT. Malar J; 2009; 8:265.

29. Wang X, Zhu S, Liu Q, Hu A, Zan Z, Yu Q. Field evaluation of the QBC technique for rapid diagnosis of vivax malaria. Bull World Health Organ; 1996; 74(6): 599.

30. WHO. Expert Committee on Malaria. Twentieth report. Geneva, World Health Organization, 2000 in WHO Technical Report Series, No. 892

31. WHO. Guidelines for the treatment of malaria, second edition. World Health Organization, Geneva. March 2010.

32. WHO. Parasitological confirmation of malaria diagnosis. Report of a WHO technical consultation Geneva, 6-8 October 2009.

33. Wongsrichanalai C, Barcus MJ, Muth S, Sutamihardja A, Wernsdorfer WH. A Review of Malaria Diagnostic Tools: Microscopy and Rapid Diagnostic Test. Am J Trop Med Hyg; 2007; 77(6):119-27. 